\title{
The Onset of Deconfinement in Nuclear Collisions
}

\author{
H. Satz ${ }^{\mathrm{a} *}$ \\ ${ }^{a}$ Fakultät für Physik, Universität Bielefeld, \\ Postfach 1001 31, D-33501 Bielefeld, Germany
}

We first consider the origin of colour deconfinement, comparing in particular spontaneous symmetry breaking and percolation. Next we specify the onset of deconfinement in nuclear collisions through parton percolation, and with parameters determined at SPS energy, we give quantitative predictions for charmonium suppression at RHIC. Finally we show that the parton cascade model leads to similar predictions, starting from a microscopic description of the space-time evolution of the collision.

\section{INTRODUCTION}

The interesting new results on the threshold and the further pattern of anomalous $J / \psi$ suppression [1.2] make it appropriate to take stock of what we can say about the onset of deconfinement in nuclear collisions and what we can predict for the forthcoming RHIC experiments. The aim of this survey is therefore to formulate the conditions for deconfinement, use the SPS results to determine the crucial onset parameters, and then calculate what should be seen at RHIC.

Finite temperature QCD predicts that strongly interacting matter will undergo a transition at $T=T_{c}$; at this point, hadronic matter, as the colour insulator phase of QCD, turns into the colour-conducting quark-gluon plasma. To throw some light on how and why this happens, we begin with a study of physical patterns for deconfinement.

\subsection{Spontaneous Symmetry Breaking vs. Cluster Percolation}

For an infinite bare quark mass, the QCD Lagrangian leads to pure colour $S U(N)$ gauge theory. Here the expectation value of the Polyakov loop $L(T)$ provides an order parameter suitable to define the confinement-deconfinement phase transition, driven by the underlying center $Z_{N} \subset S U(N)$ symmetry of the Lagrangian [3, [4]. Confined states share this symmetry, but it is spontaneously broken in the deconfined phase. Spin systems, in particular the $N$-state Potts model $(N=2$ is the Ising model), show the same kind of critical behaviour, based on the spontaneous breaking of a global $Z_{N}$ symmetry, with the 'magnetisation' $m(T)$ as order parameter. This similarity has led to the conjecture that the two systems are in fact in the same universality class of critical behaviour [5], which is indeed nicely confirmed in lattice studies [6].

${ }^{*}$ Different parts of the material presented here are based on joint work with S. Fortunato, H.-W. Huang, D. Kharzeev, M. Nardi and D. Srivastava. 
To arrive at an alternative physical definition of deconfinement, we will make use of the relation between spin and gauge systems. The critical behaviour of the Ising model can today be characterized either as

- spontaneous magnetisation, i.e., as a disorder/order transition connected to the spontaneous $Z_{2}$ symmetry breaking for $T \leq T_{c}$, or as

- percolation of clusters of parallel spins joined by suitable bonds, i.e., in terms of the geometric onset of the new phase.

These two descriptions have been shown to be completely equivalent, leading to the same critical temperature and the same critical exponents [7],8].

Since the percolation approach to the critical behaviour of spin systems is fairly new, we briefly sketch its basic features. For simplicity, consider a two-dimensional square lattice of linear size $R$; on the $R^{2}$ sites, we distribute an equal number of up and down spins according to the Ising Hamiltonian $H_{I}(T)$. With decreasing temperature $T$, adjacent sites of parallel spins will begin to form growing clusters, and for $T \leq T_{c}$, either the up or the down spins will percolate, i.e., form an infinite cluster in the thermodynamic limit $R \rightarrow \infty$. We thus can define the percolation strength

$$
P(T) \sim\left(1-\frac{T}{T_{c}}\right)^{\beta_{p}}, \quad T \leq T_{c},
$$

which measures the probability that an arbitrary site belongs to the infinite cluster. Since $P(T)=0$ for all $T \geq T_{c}$ and non-zero for all $T<T_{c}$, it constitutes an order parameter for percolation. Although the critical temperature $T_{c}$ for this transition coincides (in two dimensions) with the Curie temperature, the critical exponents in general do not. To achieve complete equivalence, including identical critical exponents, the definition of cluster has to be modified such that percolation clusters coincide with the correlation clusters obtained in the Ising model [7]. This is achieved by assigning to pairs of adjacent aligned spins in a geometric cluster an additional bond correlation, randomly distributed with the density

$$
n_{b}=1-e^{-2 J / k T},
$$

where $2 J$ just corresponds to the energy required for flipping an aligned into a nonaligned spin. The modified percolation clusters then consist of aligned spins which are bond connected. Only for $T=0$ are all aligned spins bonded; for $T>0$, some aligned spins in a purely geometric cluster are not bonded and hence do not belong to the modified cluster. This effectively reduces the size of a given cluster of aligned spins or even cuts it into several modified clusters. With such a cluster definition, cluster percolation and spontaneous magnetisation become fully equivalent as critical phenomena.

We now want to extend the percolation definition of critical behaviour to deconfinement in $S U(2)$ gauge theory, where we consider clusters of positive (or negative) Polyakov loops $L_{i}$ instead of up or down spin clusters; here $i$ denotes the space position of the Polyakov loop. First studies have been carried out in two space dimensions [9], using

$$
n_{b}=1-e^{-2 \kappa L_{i} L_{j}}
$$


as bond weight between the (same-sign) Polyakov loops $L_{i}$ and $L_{j}$ on adjacent spatial lattice sites $i$ and $j$. The effective coupling $\kappa$ is known for small temporal lattices [10]; we therefore employ for our first study $N_{\tau}=2$. Using finite size scaling to extrapolate to the thermodynamic limit of large systems, our study provides two main results:

- the percolation coupling ("temperature") coincides with the deconfinement value obtained using the expectation value of the Polyakov loop as order parameter; and

- the critical exponents for percolation strength and cluster size coincide with the Ising values predicted by the Svetitsky-Yaffe conjecture for $S U(2)$ thermodynamics.

For the specific system studied $\left[S U(2)\right.$ gauge theory, two space dimensions, $\left.N_{\tau}=2\right]$, we thus find that deconfinement can be specified in terms of Polyakov loop percolation [9] with as good or better precision than through the spontaneous symmetry breaking measured by Polyakov loop expectation values [3,4]. The next steps in this program are quite clear - one has to extend $N_{\tau}$ to arbitrary values and consider also systems in three space dimensions; then the entire study has to be repeated for $S U(3)$ gauge theory.

One particular advantage of specifying deconfinement in terms of percolation is that this appears readily generalizable to full QCD [11]. In the presence of dynamical quarks, the global $Z_{N}$ symmetry of the pure gauge theory Lagrangian is explicitly broken, so that in full QCD the expectation value of the Polyakov loop no longer serves as an order parameter for a transition - it is always non-vanishing, even though it continues to vary rapidly in a narrow temperature range. In contrast, the percolation strength $P(T)$ remains an order parameter (for the percolation transition) even for finite quark mass $m_{q}<\infty$. This situation corresponds to the Ising model with non-vanishing external field $H$; here there is also no more spontaneous symmetry breaking, with the magnetisation $m(T, H \neq 0) \neq 0$ for all $T$. Nevertheless, the percolation transition persists for all values of the temperature, tracing a curve $T_{p}(H)$ of critical temperature (the so-called 'Kertész line' $[8,12]$ ) in the $T-H$ plane. Its counterpart in QCD defines the deconfinement diagram shown in Fig. 1, separating the confined and the deconfined phase by a line of genuine (percolation) critical behaviour, not just by a rapid cross-over. It is obviously of great interest to see if this transition occurs at those temperature values where the expectation value of the Polyakov loop shows its rapid variation. Moreover, the relation between the percolation transition and the chiral transition at $m_{q}=0$ poses an interesting open problem.

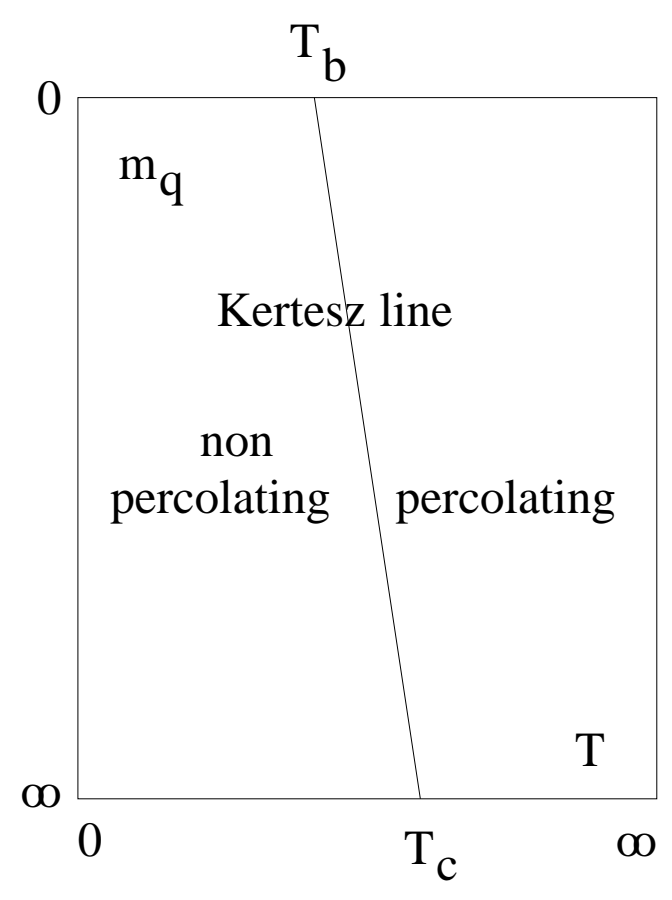

Figure 1. The Kertész line of QCD 


\subsection{Colour Screening and Quarkonium Suppression}

In media so dense that several hadrons overlap, the 'other' colour charges screen the binding forces between the quarks of any given hadron. As a result, the constituents of the medium become deconfined, since screening suppresses the long-range confining potential

$$
\sigma r \rightarrow \sigma r\left(\frac{1-e^{-\mu r}}{\mu r}\right),
$$

in a form similar to the Debye screening of the Coulomb potential. The screening mass $\mu$ is the inverse of the screening radius; it increases with temperature, so that the range of the potential decreases, and eventually deconfinement sets in. Finite temperature lattice QCD determines the critical temperature for deconfinement; it also indicates that $\mu_{c} \equiv \mu\left(T_{c}\right) \sim 0.4-0.6 \mathrm{GeV}$. With $0.3-0.5 \mathrm{fm}$, the critical colour screening radius is therefore considerably smaller than the separation between quarks inside a hadron (about $1.5-2 \mathrm{fm})$.

Colour screening provides a probe of the quark-gluon plasma [13]. Sufficiently small bound states will not melt directly at the deconfinement point; they require a shorter screening radius and hence a hotter quark-gluon plasma to be dissociated [14, 15]. Hence quarkonia can be used to to measure the temperature of deconfining media. As example, consider charmonium dissociation. The screening masses for the dissociation of the $\chi(3.5)$ and the $J / \psi(3.1)$ are about $0.35 \mathrm{GeV}$ and $0.70 \mathrm{GeV}$, respectively [14]. The $\chi$ thus melts essentially at deconfinement, while the $J / \psi$ requires a somewhat higher temperature. Similar arguments show that the $\Upsilon$ melts at a very much higher temperature. For quarkonium states in a uniform medium we thus obtain a hierarchy of suppression.

Summarizing the first section of this survey, we note

- percolation provides a natural specification and a prospective general order parameter for the onset of deconfinement;

- the dissociation of quarkonia by colour screening signals deconfinement and serves as thermometer of the deconfined medium.

In the following section, we turn to percolation in nuclear collisions and to the resulting suppression of $\chi$ and direct $J / \psi$ production.

\section{PARTON PERCOLATION 17]}

In a high energy nuclear collision viewed in the overall center of mass, the two Lorentzcontracted nuclei quickly pass through each other. After about $1 \mathrm{fm}$ at the SPS and only about $0.1 \mathrm{fm}$ at RHIC, they have separated and left behind the partonic medium which is our potential QGP candidate. Inside this medium, primary nucleon-nucleon collisions have left probes, such as heavy quark pairs or Drell-Yan dileptons, whose fate can provide information about the state of the partonic medium.

The average number of produced partons will depend on the number of nucleon-nucleon collisions and/or the number of participating ('wounded') nucleons. Up to SPS energies, the number of wounded nucleons appears to be the main determining factor [16]. This is understandable in terms of the dilated and hence large soft parton formation time 
as seen in the rest frame of either nucleus. Interference and cancellation effects of the Landau-Pomeranchuk type thus prevent soft parton emission at each nucleon-nucleon collision. At higher energies, large additional contributions can come from hard partons (minijets or jets) with short formation time, and these will be proportional to the number of collisions. Even at SPS energy, nucleon stopping and secondary multiplicities at midrapidity seem to increase somewhat with increasing mass number $A$, and this could be the onset of collision dependent effects. The $s$-dependence of the mid-rapidity multiplicity is presumably a combination of the $s$-dependence of the parton distribution function and the onset of significant hard hadron production. We shall try to include these dependences here in rather phenomenological terms, without specifying their origin.

The number of gluons of transverse momentum $k_{T}$ emitted by a wounded nucleon can be estimated using the nucleonic gluon distribution function $g(x)$ from deep inelastic scattering studies,

$$
\left(\frac{d N_{g}}{d y d k_{T}^{2}}\right)_{y=0} \simeq x g(x) f\left(k_{T}^{2}\right)
$$

where we have assumed factorization of the parton distribution in $x$ and $k_{T}$, and taken $f\left(k_{T}^{2}\right)$ as normalized to unity. At $y=0, x \simeq k_{T} / \sqrt{s}$, where $\sqrt{s}$ is the incident collision energy. Using the MRS-H form of the gluon distribution function 19 and integrating the resulting Eq. (5) over $k_{T}$ then gives us $(d N / d y)_{y=0} \simeq 2$ for SPS and 4 for RHIC energy.

Each parton of transverse momentum $k_{T}$ has an effective transverse size $r \simeq k_{T}^{-1}$, and nucleus-nucleus collisions provide many such partons overlapping in the transverse plane. We want to study their percolation behaviour. To simplify matters, we shall consider 'average' partons (rather than averaging results with $f\left(k_{T}^{2}\right)$ ). From the transverse momentum dependence of Drell-Yan dilepton production through quark-antiquark annihilation, or from that of $J / \psi$ production through gluon fusion, we know that the effective intrinsic transverse momentum of partons is $\left\langle k_{T}\right\rangle \simeq 0.75 \mathrm{GeV} / \mathrm{c}$, leading to an average transverse parton radius $r \simeq 0.27 \mathrm{fm}$. We now consider the percolation pattern for discs of that size.

As a prelude, we assume $N$ discs of radius $r$ to be randomly distributed on a flat surface of radius $R>>r$ and study the average cluster density $n_{\mathrm{cl}}(n)$ and the average cluster size $S_{\mathrm{cl}}(n)$ as a function of the overall density $n=N / \pi R^{2}$. The result is shown in Fig. 2, where we have normalized all quantities to the inverse disc area $1 / \pi r^{2}$ in order to make them dimensionless. We note that the derivative of the cluster density peaks sharply at a certain density; in the 'thermodynamic' limit $R \rightarrow \infty$, it diverges at the percolation point $n_{p}=1.175$ [20]. We see in particular that if we want to reach a certain cluster density $n_{\mathrm{cl}}$, attained at a certain overall $n$, then at this point the average cluster $S_{\mathrm{cl}}(n)$ also has a certain finite size. In other words, requiring a specific density for the onset of a new phase implies that this onset occurs for a specific finite fractional size of the system. The percolation point, for example, is reached when about $50 \%$ of the surface is covered by discs.

Turning now to nuclear collisions, we have to distribute the partonic discs not on a flat surface, but instead in the transverse plane according to the nucleon distribution determined by the profiles of the colliding nuclei [21]. Moreover, we have to allow collisions at different impact parameters, and we want to consider different $A-B$ collisions. 

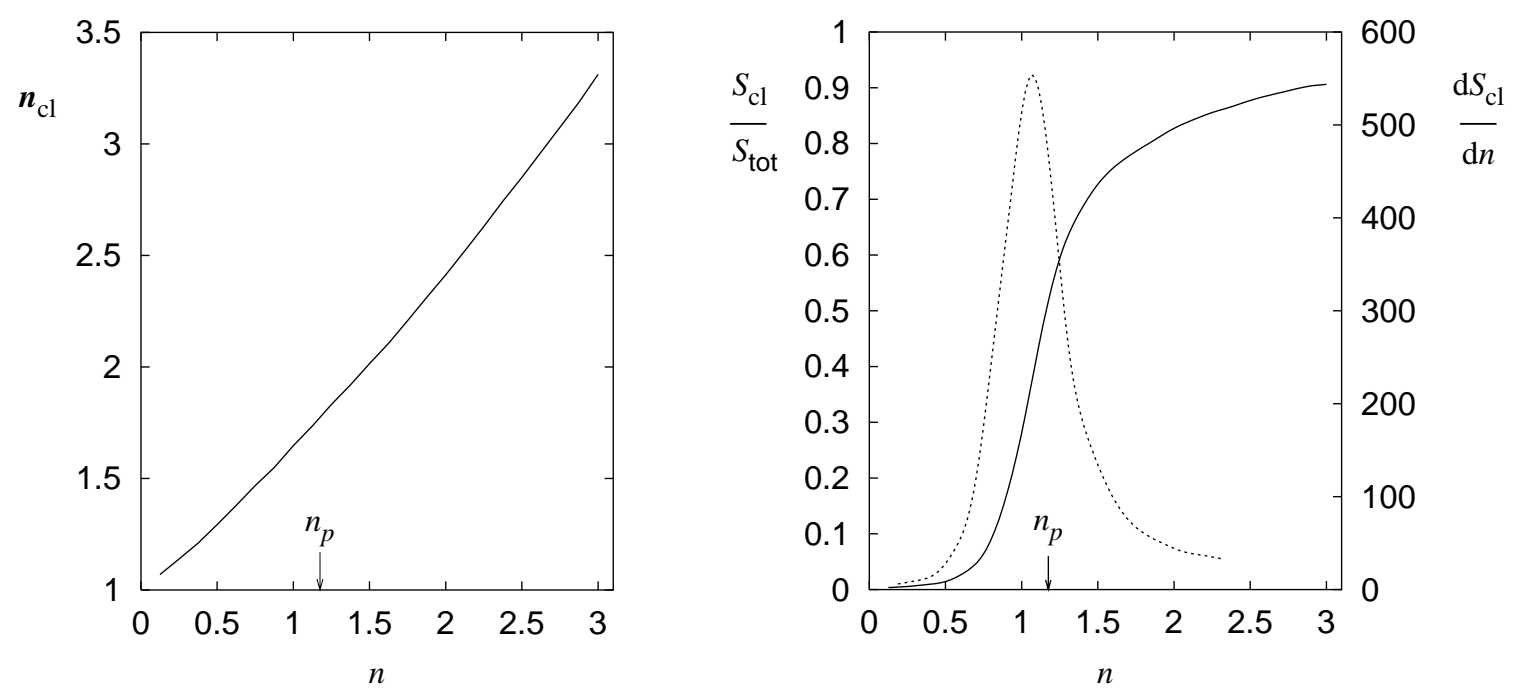

Figure 2. Average cluster density $n_{\mathrm{cl}}(n)$ (left) and average fractional cluster size $S_{\text {cl }}(n) / S_{\text {total }}$ (right) as function of the overall density $n$ of discs, for $r / R=1 / 20$; in (b), the derivative of $S_{\mathrm{cl}}(n) / S_{\text {total }}$ with respect to $n$ is also shown (dotted line). The percolation point in the limit $r / R \rightarrow 0$ is indicated by $n_{p}$.

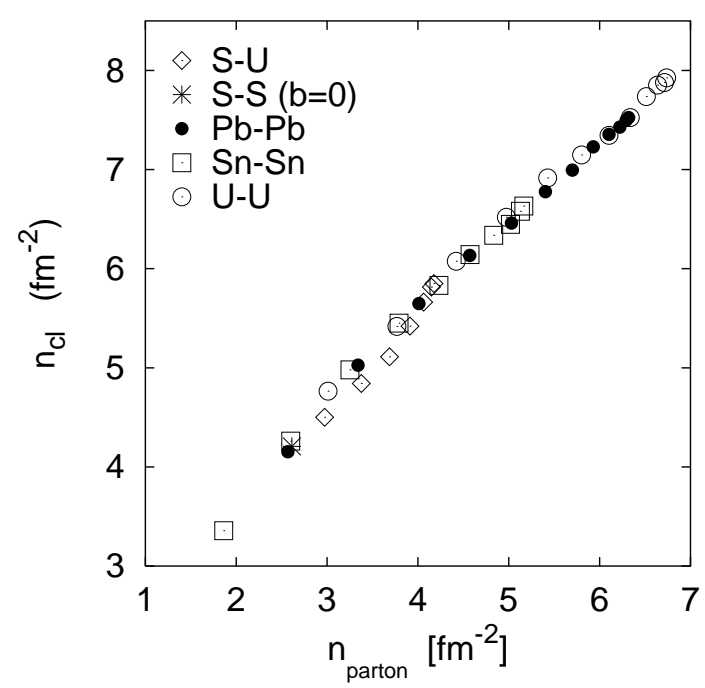

Figure 3. Cluster density vs. parton density for different centralities and different $A-B$ configurations at SPS energy.



Figure 4. Fractional cluster size vs. parton density, together with its derivative, for $\mathrm{Pb}-\mathrm{Pb}$ collisions at SPS energy. 


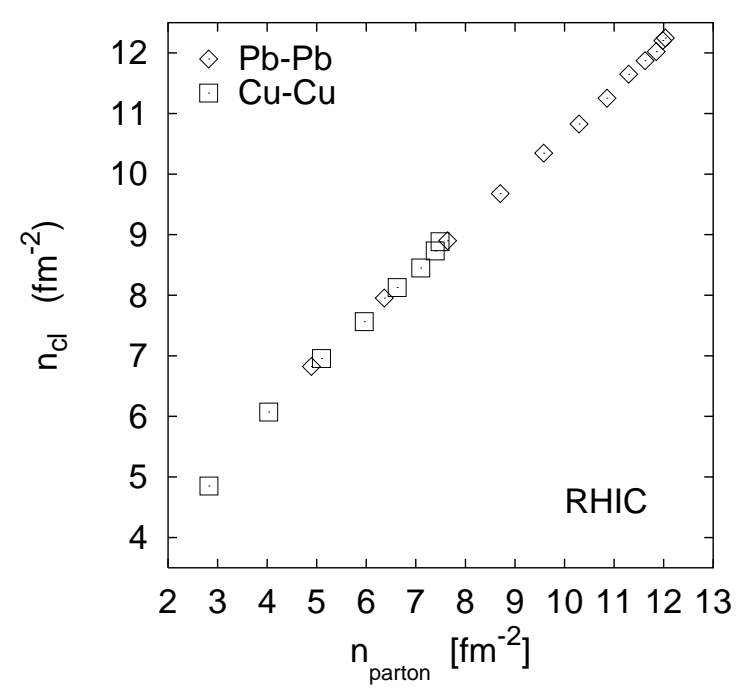

Figure 5. Cluster density vs. parton density for different centralities and different $A-B$ configurations at RHIC energy.

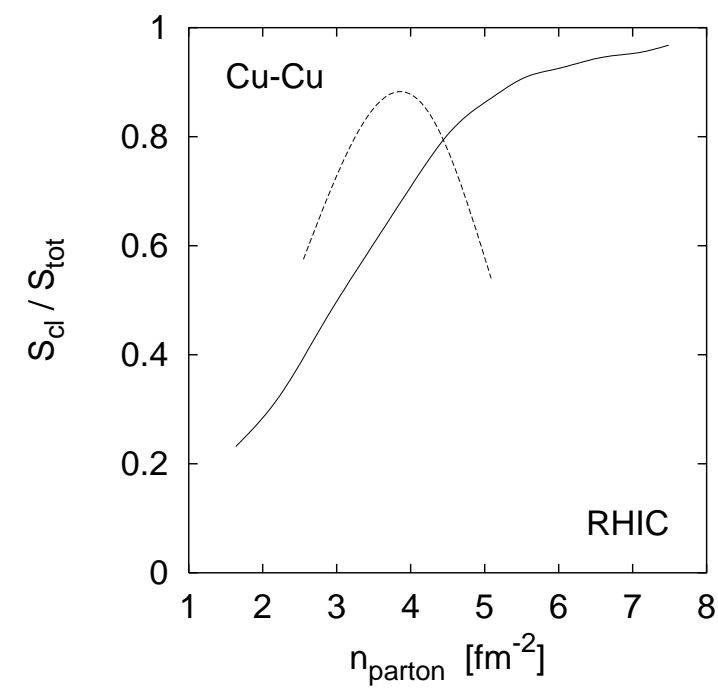

Figure 6. Fractional cluster size vs. parton density, together with its derivative, for $\mathrm{Cu}-\mathrm{Cu}$ collisions at RHIC energy.

In Fig. [3, the cluster density $n_{\mathrm{cl}}$ is shown as function of the overall density $n_{\text {parton }}$ of 'average' partons of radius $r=0.27 \mathrm{fm}$, for different centralities of various $A-B$ combinations, all at SPS energy, where we take 2 partons per wounded nucleon. For each $A-B$, the point at highest $n_{\text {parton }}$ corresponds to central (impact parameter $b=0$ ) collisions, the one at lowest $n_{\text {parton }}$ to the most peripheral collisions possible. We note that by varying $A-B$ and centrality we produce an essentially universal cluster density $n_{\mathrm{cl}}\left(n_{\mathrm{parton}}\right)$. Now we assume that deconfinement occurs at the percolation point, which can be determined by studying the average fractional cluster size $S_{\mathrm{cl}} / S_{\text {total }}$. In Fig. 团, we see the result for $P b-P b$ collisions. At the density for which the derivative peaks, $n_{\text {parton }} \simeq 4.2 \mathrm{fm}^{-2}$, the cluster density reaches its critical value $n_{\mathrm{cl}} \simeq 6 \mathrm{fm}^{-2}$. The same critical value is found for $S n-S n$ and $U-U$ collisions at SPS energy, even though this value is attained at quite different centrality in the different configurations $(b \simeq 4.5 \mathrm{fm}$ for $S n-S n, 8.5 \mathrm{fm}$ for $P b-P b$ and $10 \mathrm{fm}$ for $U-U$ collisions).

In Fig's. 5 and 6, we show the result of this procedure for RHIC energy, where we have 4 gluons per wounded nucleon. Again the critical cluster density at deconfinement is found to be $n_{\mathrm{cl}} \simeq 6 \mathrm{fm}^{-2}$. Here we have to consider $C u-C u$ collisions in order to study the onset of deconfinement, since for $P b-P b$ all possible centralities are above the threshold.

We thus conclude that the critical cluster density is, as expected, a universal quantity, independent of the choice of $A-B$, of centrality, and of incident energy. The fractional size of the deconfined cluster at threshold does, however, depend on the collision configuration: for $P b-P b$ at SPS, we have $S_{\mathrm{cl}} / S_{\text {total }} \simeq 0.45$, while $C u-C u$ at RHIC gives 0.70 . In other words, at the higher RHIC energy, deconfinement sets in for a larger bubble than at the lower SPS energy.

We further note that at SPS energy, $S-S$ and $S-U$ are below the deconfinement threshold even for the most central collisions, while at RHIC even the most peripheral 


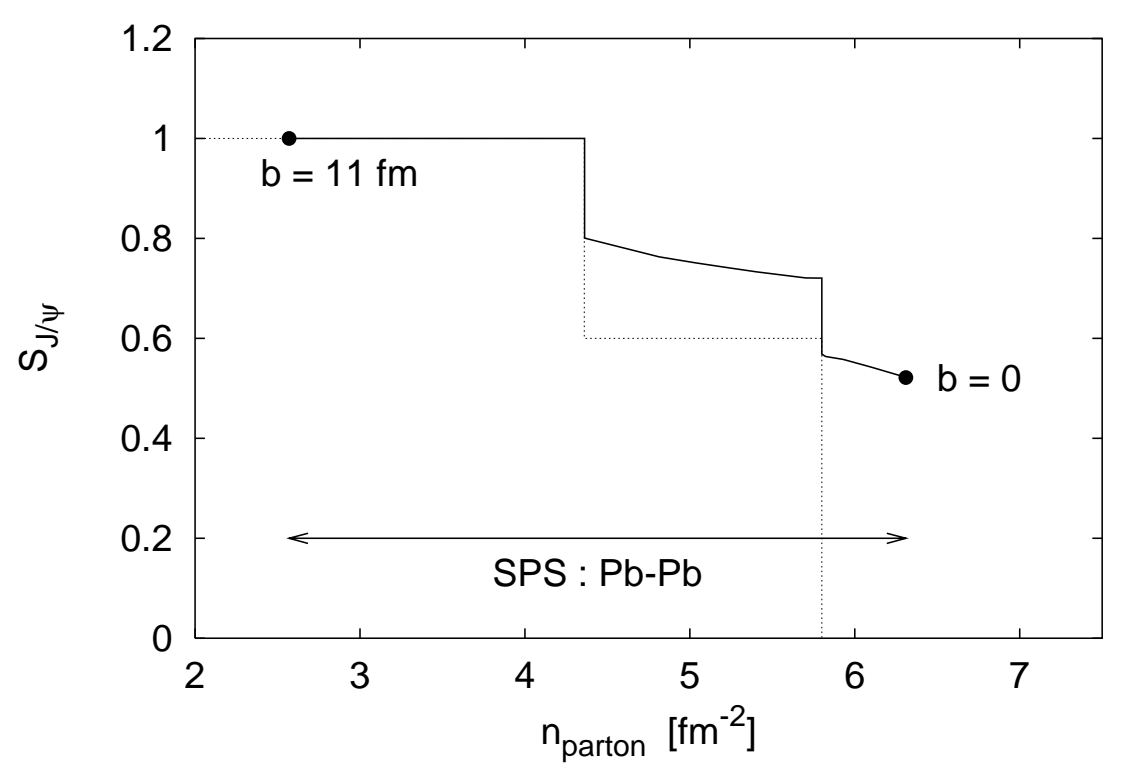

Figure 7. The $J / \psi$ survival probability as function of the parton density for $\mathrm{Pb}-\mathrm{Pb}$ collisions at SPS energy; the dotted line corresponds to a medium of uniform parton density, the solid line to collisions with parton densities determined by the profiles of the colliding nuclei.

$\mathrm{Pb}-\mathrm{Pb}$ collisions are above the threshold. To study the threshold, we thus require heavy nuclei (such as $\mathrm{Pb}$ ) at the SPS, lighter nuclei (such as $\mathrm{Cu}$ ) or lower incident energy at RHIC.

Having specified the onset of deconfinement through percolation, we now turn to deconfinement signatures. As noted above, the $\chi$ is dissociated essentially at deconfinement, so when the cluster density reaches $n_{\mathrm{cl}}^{\text {crit }} \simeq 6 \mathrm{fm}^{-2}$, all $\chi$ 's which are inside the percolating cluster disappear. The amount of $\chi$ suppression thus depends on the fractional cluster size at deconfinement, which is about $45 \%$ for $\mathrm{Pb}-\mathrm{Pb}$ at the SPS, about $70 \%$ for $\mathrm{Cu}-\mathrm{Cu}$ at RHIC and effectively $100 \%$ for $\mathrm{Pb}-\mathrm{Pb}$ at RHIC. At deconfinement, the corresponding fractions of $J / \psi$ production through intermediate $\chi$ states should thus be suppressed.

As already noted, the dissociation of directly produced $J / \psi$ 's requires a a larger screening mass and thus higher density than available at the point of deconfinement. On a microscopic level, $J / \psi$ break-up becomes possible only for harder than the average gluons at deconfinement. In principle, the relevant quantities can be determined by lattice QCD studies; however, this requires computer capabilities which are being reached only now. For the time, we therefore choose $r_{J / \psi}=0.22 \mathrm{fm}$ and $n_{\mathrm{cl}}(J / \psi)=7.8 \mathrm{fm}^{-2}$; this corresponds to a ratio of transverse dissociation energy densities $\epsilon_{T}(J / \psi) / \epsilon_{T}(\chi) \simeq 1.6$, in accord in studies combining potential theory and lattice results 14, 15. With these parameters, one obtains $20 \%$ direct $\mathrm{J} / \psi$ suppression in $\mathrm{Pb}-\mathrm{Pb}$ collisions at SPS energy, once the required cluster density is reached. The corresponding fractions for RHIC are $30 \%$ for $\mathrm{Cu}-\mathrm{Cu}$ and $35 \%$ for $\mathrm{Pb}-\mathrm{Pb}$ collisions.

Schematically, one thus obtains a suppression pattern as illustrated in Fig. 7, where it is assumed that $40 \%$ of the observed $J / \psi$ 's come from $\chi$ decay and $60 \%$ are produced 

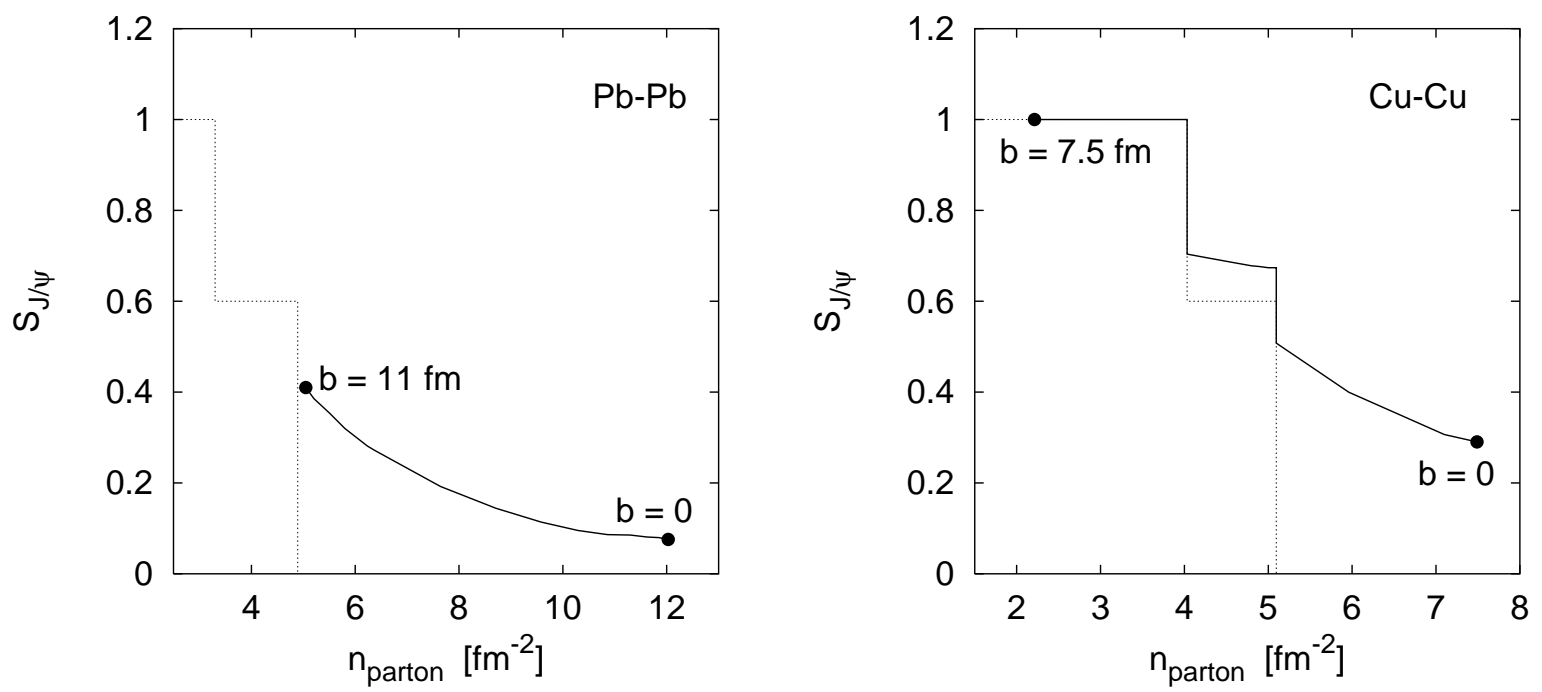

Figure 8 . The $J / \psi$ survival probability as function of the parton density for $P b-P b$ (left) and for $\mathrm{Cu}-\mathrm{Cu}$ (right) collisions at RHIC energy; the dotted line corresponds to a medium of uniform parton density, the solid line to collisions with parton densities determined by the profiles of the colliding nuclei.

directly. For clarity purposes, we ignore here the small fraction (about 8\%) coming from $\psi^{\prime}$ decay. The dotted curve corresponds to the suppression which would take place in a uniform medium of precisely specified parton density: at the deconfinement point, all $\chi$ 's are dissociated, so that the corresponding fraction of decay $J / \psi$ 's is gone; when the density for direct $J / \psi$ melting is reached, these disappear as well, leading to complete $J / \psi$ suppression. In actual nuclear collisions, the medium is not uniform, with denser 'inner' and less dense 'outer' regions in the transverse plane. Suppression now occurs only in the fraction $S_{\chi} / S_{\text {total }}$ for the $\chi$ part and $S_{J / \psi} / S_{\text {total }}$ for the direct $J / \psi$ part, where $S_{\chi}$ and $S_{J / \psi}$ denote the clusters in which the respective dissociation density is reached. Since these fractions depend on energy and nuclear geometry, the suppression curves are different for different experimental configurations. In Fig. 7, the result is shown for $\mathrm{Pb}-\mathrm{Pb}$ collisions at the SPS, from impact parameter $b=11 \mathrm{fm}$ to $b=0 \mathrm{fm}$. The difference between this curve and the one for a uniform medium thus reflects the surviving $J / \psi$ 's produced in the less dense outer regions.

In Fig. 8, similar calculations are shown for $\mathrm{Pb}-\mathrm{Pb}$ and $\mathrm{Cu}-\mathrm{Cu}$ at RHIC energy. Here we note in particular that $P b-P b$ collisions are for all meaningful centralities $(b \leq 11$ $\mathrm{fm})$ above both the $\chi$ and the direct $J / \psi$ threshold, so that we get a smooth anomalous suppression increasing from about $60 \%$ at $b=11 \mathrm{fm}$ to about $90 \%$ at $b=0$. Combining this suppression with the 'normal' pre-resonance absorption in nuclear matter, we thus predict for central $\mathrm{Pb}-\mathrm{Pb}$ collisions at RHIC a $\mathrm{J} / \psi$ production rate of less than $5 \%$ the corresponding unsuppressed rate (excluding possible $B$ decay contributions). It should also be noted that this result is based on twice the number of gluons per wounded nucleon at RHIC, compared to the SPS value. A larger increase, based on a possible larger hadron multiplicity at RHIC, would lead to more $J / \psi$ suppression. Similarly, we assume an 


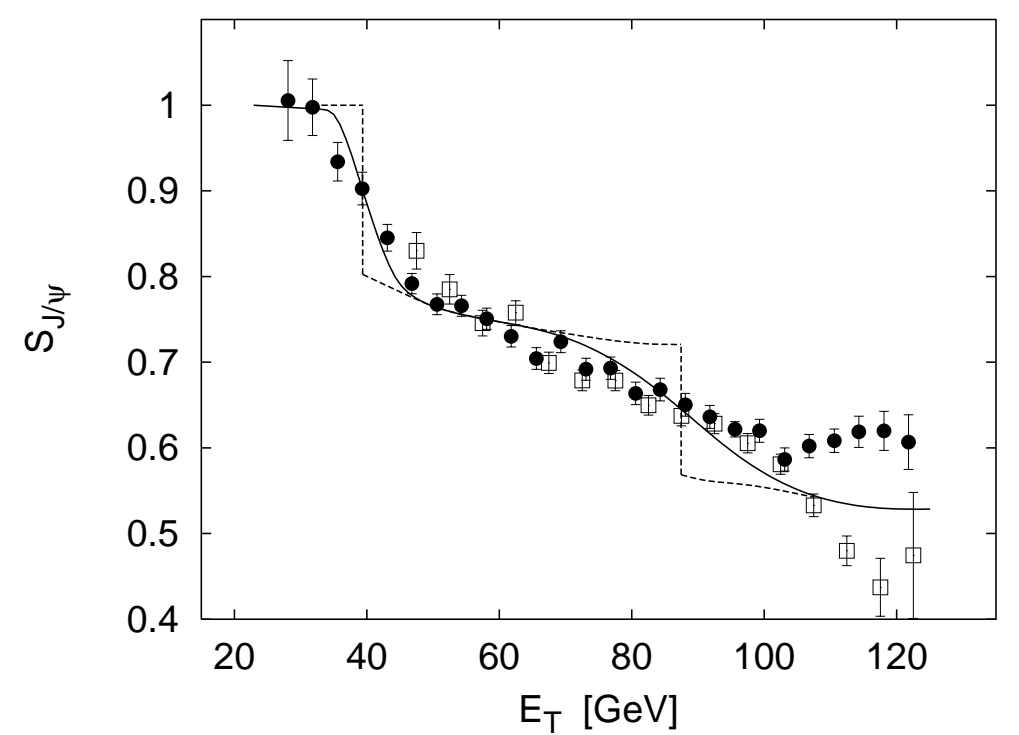

Figure 9. The $J / \psi$ survival probability as function of the measured transverse energy $E_{T}$ for $\mathrm{Pb}-\mathrm{Pb}$ collisions at SPS energy; the dashed line corresponds to collisions with fixed $E_{T}-b$ correlation, the solid line includes the experimental $E_{T}-b$ smearing. The circles show the 1997, the squares the 1998 data.

average number $\left(d N_{g} / d y\right)_{y=0}$ of gluons per wounded nucleon. It is conceivable that very central collisions reach into the tail part of the multiplicity distribution, with a larger number of hadrons and hence also gluons. This would lead to a larger suppression for very central collisions. At the SPS, the NA50 collaboration can check if the basis for this exists. By combining measurements of hadron multiplicity, transverse energy $E_{T}$ and forward energy $E_{\mathrm{ZDC}}$, it is possible to study the number of hadrons per wounded nucleon as function of $E_{T}$ and check if there is an increase at highest $E_{T}$ values.

Finally we address briefly the comparison of our results on parton percolation to the $E_{T}$-dependence of the actual data, which contain an additional smearing due to the fact that a given $E_{T}$ bin corresponds to a range of impact parameters and hence parton densities. Including this effect in the standard way [22], we obtain the result shown in Fig. 9. Included in this figure are the 1997 [24] and the 1998 data [1,2] with minimum bias determined Drell-Yan reference. The high $E_{T}$ points of the 1997 data show an enhancement due to rescattering effects, which are removed in the 1998 data using a thinner target. While reproducing the overall behaviour, our results clearly show deviations from the data in detail. As mentioned, the levelling-off of our curve at high $E_{T}$ would have to be modified if an increase in the multiplicity per wounded nucleon at high $E_{T}$ should be observed. Another possible modification could enter through a density-dependent charmonium dissociation. We have here assumed that all $\chi$ 's melt once the critical density is reached. Allowing a partial survival chance, which decreases with increasing density, would lead to a steeper drop of the suppression with $n_{\text {parton }}$ as well as with $E_{T}$. One possible source for such an effect would be the finite life-time of the deconfining medium in actual nuclear collision, as seen e.g. in the parton cascade model to be discussed in the next section. 
Summarizing this section, we note that parton percolation provides a consistent framework to study the onset of deconfinement as well as the onset of charmonium suppression as a signature of the transition. It is clear that the compatibility of the parton basis with other, soft hadron production processes has to be checked [18].

\section{COLOUR SCREENING IN THE PARTON CASCADE MODEL}

Probing deconfinement in nuclear collisions means studying the early partonic medium left behind when the Lorentz-contracted nuclei have passed through each other - a medium consisting of partons, of strings, of flux tubes, or of whatever basis is chosen. Any model of this type has to

- define deconfinement,

- specify the onset of a particular signature such as charmonium suppression, and

- check that soft hadron production is consistent with the picture.

In the past section, we had chosen a purely geometric framework, with deconfinement defined as percolation of partons of transverse radius $r \simeq 1 / k_{T}$, and with a given (energydependent) number of partons emitted from each wounded nucleon. In this section, we want to consider a more detailed microscopic model, based on the interaction of partons and the resulting evolution of the partonic medium 25]. For this, we shall use the parton cascade model [26]; but evidently one could also use other approaches, such as HIJING [27] or the dual parton model [28]. All such models provide a parton or string distribution in the transverse plane and thus in principle constitute an alternative to the nuclear profile pattern we had used in the previous section. The time evolution of such a distribution from the parton cascade model is shown in Fig. 10 and compared to the nuclear profile pattern in Fig. 11. It is seen there that the parton cascade model in fact provides a transverse parton distribution very similar to that based on the distribution of wounded nucleons.

The parton cascade model describes the partonic medium before the onset of confinement. To determine when confinement sets in for such a medium, we calculate the screening mass $\mu$ and recall that lattice $\mathrm{QCD}$ finds $\mu_{c} \simeq 0.4-0.6 \mathrm{GeV}$ at the deconfinement point. The screening mass is given by [29]

$$
\mu^{2}=-\frac{3 \alpha_{s}}{\pi^{2}} \int d^{3} k \nabla f(k),
$$

where $f(k)$ specifies the momentum distribution of the partons. For a Bose-Einstein distribution, with $f(k)=\left(1-e^{-k / T}\right)^{-1}$, Eq. (6) gives the familiar $\mu^{2}=4 \pi \alpha_{s} T^{2}$ obtained in perturbative QCD.

Instead, we now insert for $f(k)$ the time-dependent distribution function $f_{\mathrm{PCM}}(k, t)$ from the parton cascade model, in order to obtain the non-equilibrium time evolution of the screening mass. First preliminary results for central $\mathrm{Pb}-\mathrm{Pb}$ collisions at RHIC are given in Fig. 12, indicating the variation of $\mu$ as function of the proper medium life-time after full nuclear overlap and as function of the transverse radius of the medium. To interpret this behaviour, recall that deconfinement as well as $\chi$ dissociation occurs for 

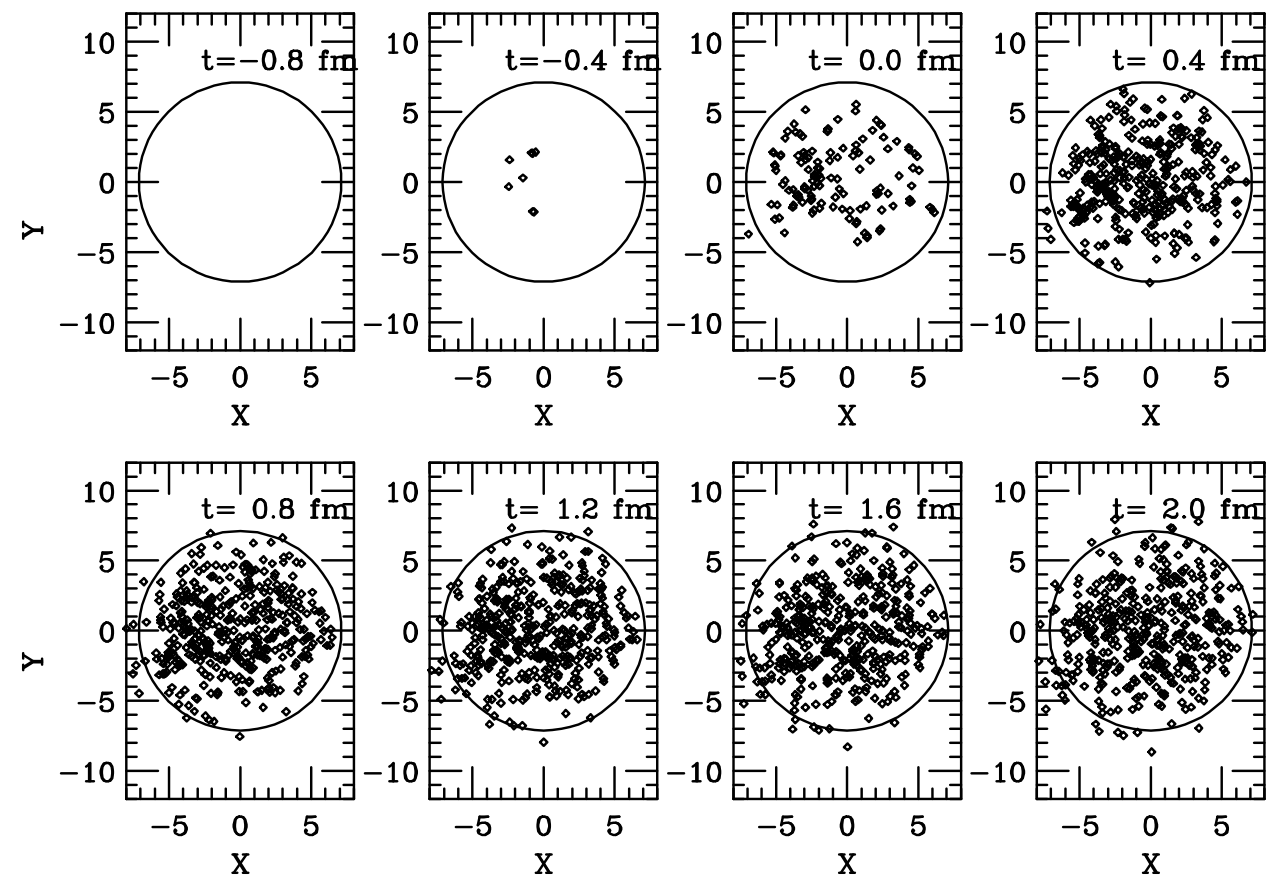

Figure 10. Preliminary PCM calculations for the time evolution of the parton population in the transverse plane for central $\mathrm{Pb}-\mathrm{Pb}$ collisions at the SPS.

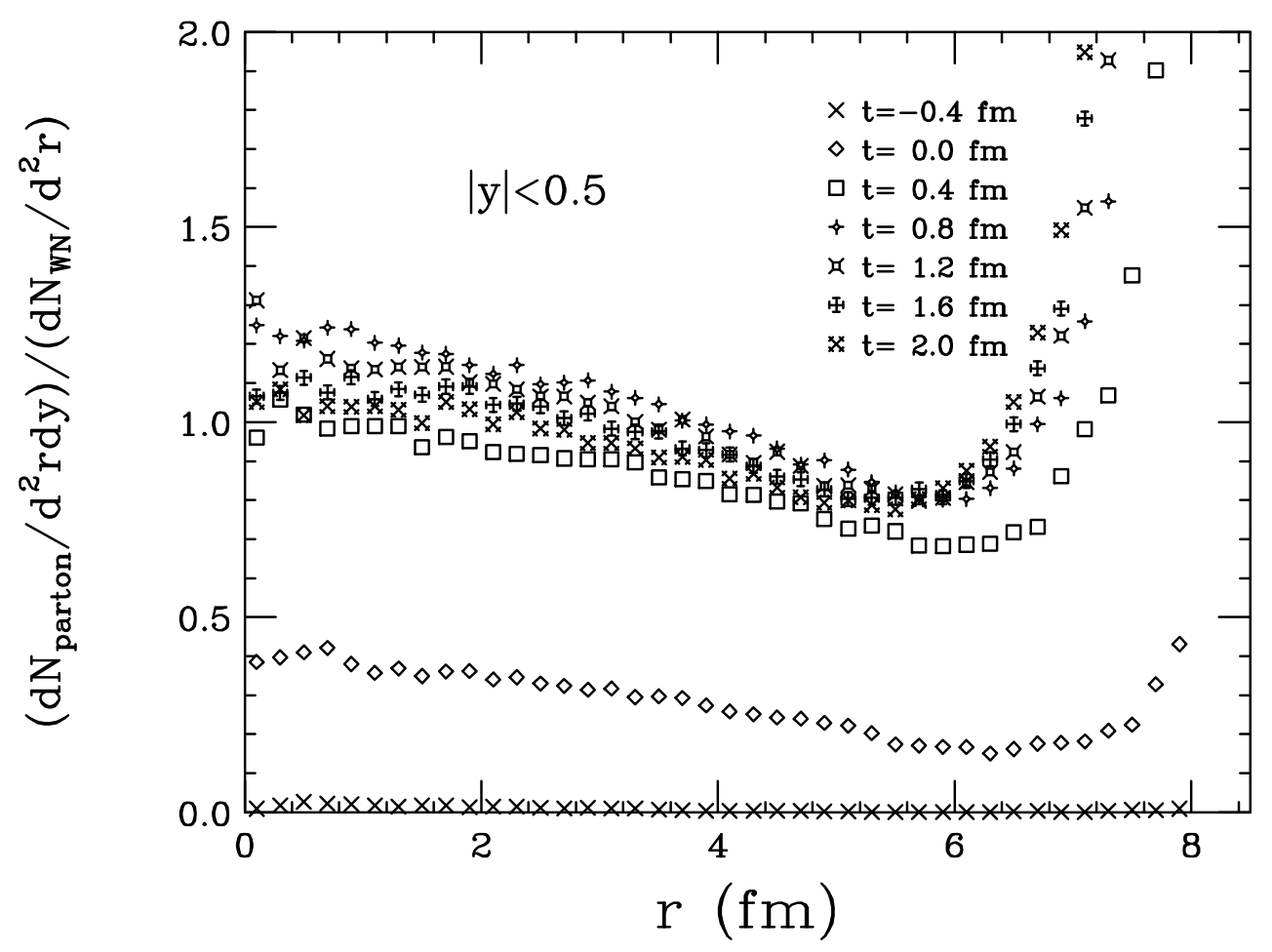

Figure 11. Preliminary PCM calculations for the radial density of the parton population in the transverse plane of central SPS $P b-P b$ collisions at different collision times, compared to that obtained from the wounded nucleon distribution. 


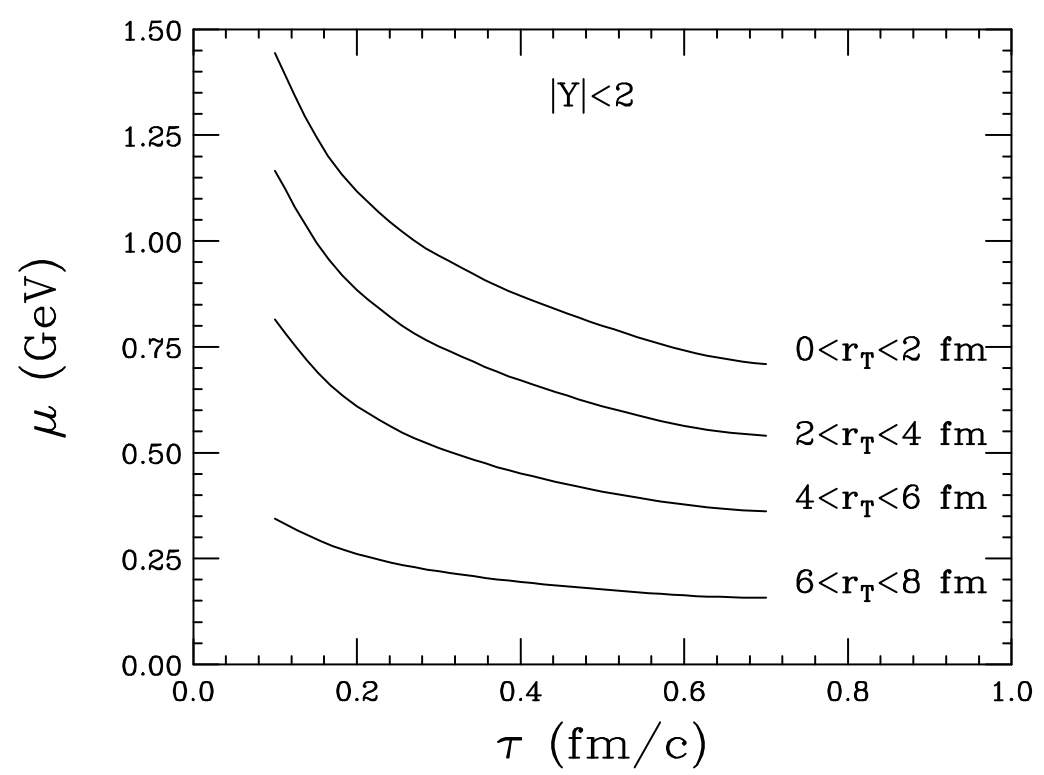

Figure 12. Preliminary PCM calculations for the time evolution of the colour screening mass in different transverse regions of a central $\mathrm{Pb}-\mathrm{Pb}$ collision at RHIC energy.

$\mu \geq 0.4-0.6 \mathrm{GeV}$, while the dissociation of directly produced $J / \psi$ 's requires $0.7 \mathrm{GeV}$. Hence for essentially all production regions, our preliminary results for the screening mass exceed the necessary dissociation values for both $\chi$ and $J / \psi$ for some time. In accord with the results from parton percolaton, this would seem to indicate almost complete $J / \psi$ suppression for central $P b-P b$ collisions at RHIC. To really conclude this, however, one must first specify how long $\mu$ has to exceed its critical value in order to 'melt' the charmonium state in question. In any case, for really quantitative precision more extensive calculations are necessary.

Let us finally note one particular advantage of microscopic evolution picture such as the parton cascade model. Once we have defined the conditions for quarkonium suppression, be it through percolation or through colour screening, the parton cascade model can be used to check if the conditions needed for the observed suppression lead to consistent soft hadron production at the later times. Such models thus provide a bridge between the early deconfined and the later hadronic stages. The situation is summarized in Fig. 13, which also serves as our conclusion. It shows on one hand the general evolution of the collision, on the other the effect of this evolution on charmonium production as hard probe of the early medium.

\section{ACKNOWLEDGEMENTS}

It is a pleasure to thank Ph. Blanchard, S. Fortunato, H.-W. Huang, F. Karsch, D. Kharzeev, M. Nardi, D. K. Srivastava and D. Stauffer for helpful comments and discussions.

\section{REFERENCES}




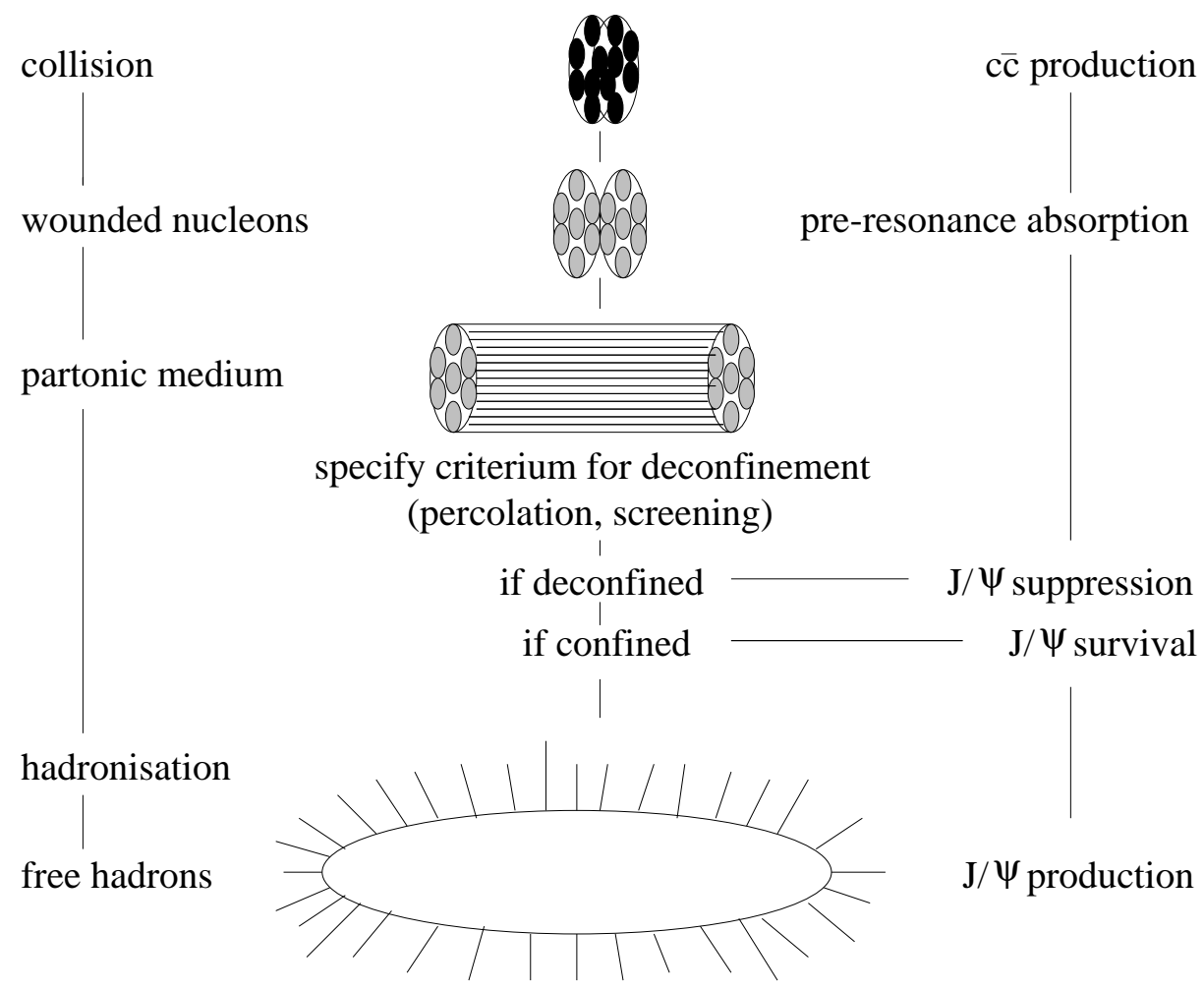

Figure 13. A short history of deconfinement

1. C. Cicalò, these proceedings.

2. L. Kluberg, these proceedings.

3. L. McLerran and B. Svetitsky, Phys. Lett. 98 B (1981) 195

4. J. Kuti, J. Polónyi and K. Szlachányi, Phys. Lett. 98 B (1981) 199.

5. B. Svetitsky and L. G. Yaffe, Nucl. Phys. B 210 [FS6] (1982) 423.

6. J. Engels et al., Phys. Lett. B 365 (1996) 219

7. A. Coniglio and W. Klein, J. Phys. A 13 (1980) 2775.

8. For a recent survey, see D. Stauffer and A. Aharony, Introduction to Percolation Theory, Taylor \& Francis, London 1994.

9. S. Fortunato, H.-W. Huang and H. Satz, in preparation;

S. Fortunato, these proceedings.

10. F. Green and F. Karsch, Nucl. Phys. B 238 (1984) 297.

11. H. Satz, Nucl. Phys. A642 (1998) 130c.

12. J. Kertész, Physica A 161 (1989) 58.

13. T. Matsui and H. Satz, Phys. Lett. 178B (1986) 416.

14. F. Karsch, M. T. Mehr and H. Satz, Z. Phys. C 37 (1988) 617.

15. F. Karsch and H. Satz, Z. Phys. C 51 (1991) 209.

16. A. Białas, M. Bleszyński and W. Czyz, Nucl. Phys. B 111 (1976) 461.

17. D. Kharzeev, M. Nardi and H. Satz, in preparation. 
18. N. Armesto et al., Phys. Rev. Lett. 77 (1996) 3736.

19. A. D. Martin, R. G. Roberts and W. J. Stirling, Int. Journal of Mod. Phys. A 10 (1995) 2885.

20. U. Alon, A. Drory and I. Balberg, Phys. Rev. A 42 (1990) 4634.

21. C. W. deJager, H. deVries and C. deVries, Atomic Data and Nuclear Data Tables 14 (1974) 485.

22. D. Kharzeev, C. Lourenço, M. Nardi and H. Satz, Z. Phys. C 74 (1997) 307.

23. M. Nardi and H. Satz, Phys. Lett. B 442 (1998) 14.

24. L. Ramello (NA50), Nucl. Phys. A 638 (1998) 261c

M. C. Abreu et al.(NA50), Phys. Lett. B450 (1999) 456.

25. D. K. Srivastava and H. Satz, in preparation

26. K. Geiger and B. Müller, Nucl. Phys. B 369 (1992) 600

K. Geiger, Phys. Rep. 258 (1995) 376.

27. M. Gyulassy and X.N. Wang, Phys. Rev. D 45 (1992) 844.

28. See A. Capella et al., Phys. Rep. 236 (1994) 225.

29. T. S. Biro, B. Müller and X.-N. Wang, Phys. Lett. B 283 (1992) 171. 